\title{
Thrombelastometry guided blood- component therapy after cardiac surgery: a randomized study
}

Martin Haensig ${ }^{1 *+} \mathbb{D}$, Joerg Kempfert ${ }^{2 \dagger}$, Pia-Maria Kempfert ${ }^{2}$, Evaldas Girdauskas ${ }^{3}$, Michael Andrew Borger $^{4}$ and Sven Lehmann ${ }^{4}$

\begin{abstract}
Background: Significant bleeding is a well known complication after cardiac surgical procedures and is associated with worse outcome. Thrombelastometry $\left(\mathrm{ROTEM}^{\circledR}\right)$ allows point-of-care testing of the coagulation status but only limited data is available yet. The aim was to evaluate the ROTEM ${ }^{\oplus}$-guided blood component therapy in a randomized trial.
\end{abstract}

Methods: In case of significant postoperative bleeding (> $200 \mathrm{ml} / \mathrm{h}$ ) following elective isolated or combined cardiac surgical procedures (including 14\% re-do procedures and $4 \%$ requiring circulatory arrest) patients were randomized to either a 4-chamber ROTEM ${ }^{\circledR}$-guided blood-component transfusion protocol or received treatment guided by an algorithm based on standard coagulation testing (control). One hundred four patients (mean age: 67.2 \pm 10.4 years, mean log. EuroSCORE $7.0 \pm 8.8 \%$ ) met the inclusion criteria. Mean CPB-time was $112.1 \pm 55.1$ min., mean cross-clamp time $72.5 \pm 39.9$ min.

Results: Baseline demographics were comparable in both groups. Overall there was no significant difference in transfusion requirements regarding red blood cells, platelets, plasma, fibrinogen or pooled factors and the re-thoracotomy rate was comparable (ROTEM ${ }^{\oplus:} 29 \%$ vs. control: $25 \%$ ). However, there was a trend towards less $24-h$ drainage loss visible in the ROTEM ${ }^{\oplus}$-group (ROTEM ${ }^{\oplus:} 1599.1 \pm 834.3 \mathrm{ml}$ vs. control: $1867.4 \pm 827.4 \mathrm{ml} ; p=0.066$ ). In the subgroup of patients with long CPB-times (> 115 min.; $n=55$ ) known to exhibit an increased risk for diffuse coagulopathy ROTEM ${ }^{\oplus}$-guided treatment resulted in a significantly lower 24-h drainage loss (ROTEM ${ }^{\oplus}: 1538.2 \pm$ $806.4 \mathrm{ml}$ vs. control: $2056.8 \pm 974.5 \mathrm{ml} ; p=0.032$ ) and reduced 5-year mortality (ROTEM ${ }^{\oplus:} 0 \%$ vs. control: $15 \%$; $p=0.03)$.

Conclusion: In case of postoperative bleeding following cardiac surgical procedures a treatment algorithm based on "point-of-care" 4-chamber ROTEM ${ }^{\circledR}$ seems to be at least as effective as standard therapy. In patients with long CPB-times ROTEM ${ }^{\oplus}$-guided treatment may result in less bleeding, a marked reduction in costs and long-term mortality.

Trial registration: German Clinical Trials Register, TRN: DRKS00017367, date of registration: 05.06.2019, 'retrospectively registered'.

Keywords: Cardiac surgery, Bleeding, Point-of-care testing, Thrombelastometry, Blood-component therapy, Transfusion

\footnotetext{
* Correspondence: mhaensig@gmail.com

${ }^{\dagger}$ Martin Haensig and Joerg Kempfert contributed equally to this work.

${ }^{1}$ Department of Vascular Surgery, Cardiovascular Center, University of Leipzig,

Liebigstr 20, 04103 Leipzig, Germany

Full list of author information is available at the end of the article
}

(c) The Author(s). 2019 Open Access This article is distributed under the terms of the Creative Commons Attribution 4.0 International License (http://creativecommons.org/licenses/by/4.0/), which permits unrestricted use, distribution, and reproduction in any medium, provided you give appropriate credit to the original author(s) and the source, provide a link to the Creative Commons license, and indicate if changes were made. The Creative Commons Public Domain Dedication waiver (http://creativecommons.org/publicdomain/zero/1.0/) applies to the data made available in this article, unless otherwise stated. 


\section{Background}

Postoperative impairment of hemostasis is a well known phenomenon associated with cardiac surgical procedures due to the operative trauma, cardiopulmonary bypass, heparinisation and subsequent reversal and other factors $[1,2]$. Significant postoperative bleeding has to be expected in $5-10 \%$ of patients $[3,4]$ and is associated with significantly worse outcome and an up to 8-fold increase in mortality [4]. Although it has been estimated that cardiac surgery accounts for $20 \%$ of all national blood products consumption in the United States [3] strategies for blood component therapy demonstrate a great variability [5]. Given the known side-effects of blood components and costs it is of concern that a substantial proportion of these products seems to be transfused unnecessarily [6]. Or in other words: the surgical center has been identified as an independent risk factor for transfusion [5].

Well-structured blood component therapy protocols based on standard coagulation testing have been proven to be beneficial in comparison to commonly used "empiric" treatment [7]. Treatment algorithm guided by "point-of-care" thrombelastometry $\left(\right.$ ROTEM $\left.^{\circ}\right)$ might be associated with further benefits as they are capable to specifically identify potential coagulation disorders in a timely manner allowing specific and fast treatment $[8$, 9]. However, for cardiac surgical patients only limited data is available from a few studies $[10,11]$.

The aim was to assess efficacy of a ROTEM $^{\circ}$-guided blood component treatment algorithm for elective cardiac surgical patients in comparison to a protocol based on standard coagulation testing in a prospective randomized trial.

\section{Methods}

\section{Study design}

After approval by the local ethics committee of the University of Leipzig (reference nr.: 049/07-ek), and written consent obtained from each patient, 104 patients scheduled for elective cardiac surgical procedures were enrolled in this single center study at the Heartcenter, Leipzig. Patients that demonstrated significant persistent postoperative bleeding defined as drainage loss of $100 \mathrm{ml} / 30 \mathrm{~min}$ or more than $200 \mathrm{ml} / \mathrm{h}$ after arrival at the intensive care unit (ICU) were randomized. The first group (ROTEM ${ }^{\circ}$ ) were treated based on a thrombelastometry-guided (4chamber ROTEM ${ }^{\mathrm{TM}}$ ) blood-component transfusion protocol whereas the other group (Control) received treatment guided by a protocol based on standard coagulation testing.

Primary endpoints were transfusion requirements regarding red blood cells, platelets, plasma, fibrinogen or pooled factors. The secondary endpoints were 24-h drainage loss, re-thoracotomy rate and cost analysis of blood and coagulation products.

During the study period of $2 \frac{1}{2}$ years, a total of 6041 patients underwent elective cardiac surgical procedures and the rethoracotomy rate was $8.3 \%$ at our center.

\section{Subgroup analysis of patients at "high-risk" for bleeding (long CPB times)}

Since patients with long CPB-times are known to exhibit an increased risk for diffuse coagulopathy, a priori a subgroup analysis regarding this specific risk factor was planned. Regarding the recent literature, an appropriate definition of the term "prolonged CPB time" is unclear $[12,13]$. Nevertheless, we choose the mark of $115 \mathrm{~min}$, because this was the mean of our CPB times based on our preliminary study results. However, and most importantly post hoc all CPB times $\geq 115$ min revealed evidence for a significant difference.

\section{Patients}

Preoperative antiplatelet therapy with clopidogrel or anticoagulation with coumadine was discontinued 7 days prior to cardiac surgery. In case of drug eluting stent implantation prior to the planned procedure, if possible surgery was defered for more than 4 weeks. Patients were eligible for inclusion in case of significant bleeding postoperatively after standard elective procedures. Patients with therapy-relevant known coagulation abnormalities (hemophilia-A /B, APC resistance, factor XIII deficiency, HIT-II etc.) were not included. In addition, patients that suffered live-threatening bleeding requiring immediate re-thoracotomy, mass-transfusion or ECMO support were excluded, as these cases usually are associated with multiple confounding variables that might not allow for a valid comparison of groups.

\section{Rotational 4-chamber Thromboelastometry (ROTEM ${ }^{\circledR}$ )}

The ROTEM ${ }^{\circledR}$ (Pentapharm GmbH, Munich, Germany) system allows for "point-of-care "coagulation testing based on the classic thrombelastometry. Basic principles of the ROTEM $^{\ominus}$ have been described in detail elsewhere [14]. Briefly, blood samples are activated and the time until first clot formation is measured (clotting time - CT). In addition maxium clot firmness (MCF) is assessed. The CT is mainly dependent on the availability of "intrinsic" and/or "extrinsic" coagulation factors and Heparin action, while the MCF depends on platelets function and fibrinogen. The ROTEM $^{\circledR}$ system allows for four simultaneous measurements in separated chambers thus assessing the patient's complete coagulation status with one run. Within the trial protocol the four chambers were utilized as follows: (1) INTEM: intrinsic pathway activation (2) HEPTEM: intrinsic activation, Heparin deactivated by Heparinase (3) FIBTEM: extrinsic activation, platelets inactivated (4) APTEM: 
extrinsic activation, added aprotinin. In addition, EXTEM (extrinsic pathway activation) was used in case of a reasonable suspicion for factor-VII deficiency.

\section{Intraoperative care}

Surgery was performed according to clinical standards. In case serum calcium and $\mathrm{pH}$ were not within the standard range they were adjusted according to our internal standard operating procedures. During $\mathrm{CPB}$ aprotinin was administred with a loading dose of 1 to 2 Million IU followed by 0.5 Million IU per hour maintenance dose. Within the duration of the trial it has been withdrawn from the marked and was replaced by tranexamic acid. Tranexamic acid was administred with a loading dose of $10 \mathrm{mg} / \mathrm{kg}$ bodyweight over $20 \mathrm{~min}$ followed by a $1 \mathrm{mg} / \mathrm{kg}$ bodyweight/h infusion.

Special attention was applied to sufficient rewarming on cardiopulmonary bypass aiming at a body temperature (bladder) of $37^{\circ} \mathrm{C}$. After Heparin reversal, guided by repeat ACT measurements, coagulation products were administered in case of visible diffuse bleeding assessed by the surgeon on an empirical basis until standard coagulation test results were available. All patients received the same intraoperative care independent of their further course and potential randomization.

\section{Management in the ICU}

After the procedure all patients were transferred to the intensive care unit. Extubation was considered as early as possible using a "fast-track" protocol. Arterial blood gas analyses were performed on a regular basis. Immediately after arrival at the ICU routine blood samples were sent to the laboratory including baseline coagulation testing (PTT, Quick, platelet count) in all patients according to routine practice. In case of significant bleeding (see definition above) patients were randomized to either ROTEM $^{\circ}$ or standard coagulation-test guided treatment using a computer-generated randomization list. The allocation sequence was concealed from the researcher (PMK) enrolling and assessing participants in sequentially numbered, opaque, sealed and stapled envelopes. A thin aluminium foil inside the envelope was used to render the envelope impermeable to intense light. Corresponding envelopes were opened only after the enrolled participants completed all baseline assessments and it was time to allocate the intervention (JK, EG). Randomization was performed immediately after arrival at the intensive care unit, because conventional laboratory test are usually not available on time in the operating room and furthermore comparison to a wellstructured standard algorithm would then not have been possible. The allocation list was stored on a separate folder in the Coordinating Centre of Clinical Trials at the Heartcenter Leipzig.
For patients in the ROTEM $^{\circ}$-group a four-chamber analysis was performed as described above. Further blood component therapy was performed according to the ROTEM $^{\circ}$-guided protocol (Table 1). In patients randomized into the control group further "extended" standard coagulation test were sent to the laboratory and treatment was guided by an algorithm based on standard coagulation tests (Table 2).

The decision to perform a re-thoracotomy was made by the surgeon in charge of the patient independent of the respective group randomization.

Packed red blood cells (RBCs) were transfused triggered only by a haemoglobin level of less than $8 \mathrm{~g} / \mathrm{dl}$ in the arterial blood-gas analysis. Preload (transfusion of crystalloid volume) was maintained liberally to avoid vasopressor therapy as far as possible. Care was taken to maintain sufficient body temperature by transfusing prewarmed fluids and using forced-air warming blankets.

\section{Follow-up, data analysis and statistics}

A power analysis was based on our initial experience with the $\mathrm{ROTEM}^{\circ}$ analyzer and the results from previous studies using thrombelastography-guided coagulation management in cardiac surgery $[11,15]$. The power analysis suggested that 30 patients per group would be required to demonstrate a $40 \%$ reduction in the use of allogeneic blood products with $\alpha$ of 0.05 and power of $80 \%$.

Four patients were considered protocol violators, consequently 104 patients remained for the per-protocol analyses. Data were collected prospectively and 30-day follow-up was $100 \%$ complete. All statistical analyses were performed using SPSS, version 24.0 (Chicago, IL, USA). Continuous variables are expressed as mean \pm standard deviation for Gaussian distributed variables and otherwise median values. For comparison of continuous variables the two-tailed Student's t-test and, for nonnormally distributed variables, the Mann-Whitney Utest were utilized. A $p$-value of less than 0.05 was considered statistically significant. The person performing data analysis and statistical calculations was blinded for the treatment modality. The study adheres to the current version of the CONSORT guidelines (Additional files 1 and 2) [16].

\section{Results}

Between 03/2007 and 08/2009 104 patients met the inclusion criteria. Mean age was $67.2 \pm 10.4$ years, mean $\log$ EuroSCORE (European System for Cardiac Operative Risk Evaluation) was $7.0 \pm 8.8 \%$, and Society of Thoracic Surgeons (STS) Score was $2.4 \pm 2.7 \%$. Fifty patients $(48 \%)$ received aortic valve replacement (AVR) and twenty-eight patients (26.9\%) isolated CABG, whereas $25 \%$ received AVR in combination with isolated CABG. 
Table 1 Transfusion protocol for the ROTEM ${ }^{\oplus}$-guided group

\begin{tabular}{|c|c|}
\hline Trigger: & $\begin{array}{l}\text { drainage loss }>200 \mathrm{ml} / \mathrm{h} \text { or } 100 \mathrm{ml} / 30 \mathrm{~min} \text { at } 30 \mathrm{~min} \text { after } \\
\text { arrival at the ICU }\end{array}$ \\
\hline 1. & $\begin{array}{l}\text { 4-chamber TEM ( } 1 \text { INTEM, } 2 \text { HEPTEM, } 3 \text { FIBTEM, } 4 \text { APTEM), } \\
\text { Quick, AT3 }\end{array}$ \\
\hline 2. & CT-INTEM/CT-HEPTEM $>1.5=>\mathbf{5 0 0 0}$ IE Protamin \\
\hline 3. & CT-HEPTEM $>260 \mathrm{~s}=>$ FFP units $(\mathrm{kg} \text { body weight })^{\mathrm{a}}$ \\
\hline 4. & $\begin{array}{l}\text { a: MCF-HEPTEM } 35-45 \mathrm{~mm} \text { and MCF-FIBTEM }>8 \mathrm{~mm}=>\mathbf{1} \\
\text { platelet concentrate } \\
\text { b: } M C F-H E P T E M<35 \mathrm{~mm}=>\mathbf{1} \text { platelet concentrate }\end{array}$ \\
\hline 5. & MCF-FIBTEM $<8 \mathrm{~mm}=>\mathbf{2}$ g Fibrinogen \\
\hline \multirow[t]{2}{*}{6.} & $\begin{array}{l}\text { MCF-APTEM/MCF-HEPTEM }>1.5 \text { or Aprotinin effective optically } \\
=>\mathbf{2} \text { Mio IE Aprotinin } \mathbf{2} \mathbf{~ g} \text { Tranexamic acid }\end{array}$ \\
\hline & $\begin{array}{l}\text { - In case of persistent bleeding - > re-testing according to (1) } \\
\text { and further therapy as suggested by the protocol. } \\
\cdot 2000 \text { IE PPSB if INR }>2.0 \text { and known liver dysfunction or } \\
\text { previous Coumadin therapy. } \\
\text { - } 2000 \text { IE AT3 if an increase }>50 \% \text { (prothrombin time) is not to } \\
\text { be expected via FFP-substitution. }\end{array}$ \\
\hline
\end{tabular}

Transfusion of RBCs according to the haemoglobin level of the blood-gasanalysis. Target value $>8.0 \mathrm{~g} / \mathrm{dl}$

${ }^{\mathrm{a}} \mathrm{FFP}(15 \mathrm{ml} / \mathrm{kg} \mathrm{KG}):<58 \mathrm{~kg}$ body weight - $>3 \mathrm{FFP}, 58-75 \mathrm{~kg}$ body weight - > 4 FFP, 75-92 kg body weight - > 5 FFP, $>92 \mathrm{~kg}$ body weight - > 6 FFP

The entries in boldface represent the administered blood-components

Mean CPB-time was $112.1 \pm 55.1 \mathrm{~min}$., mean cross-clamp time $72.5 \pm 39.9 \mathrm{~min}$., $13 \%$ of procedures were re-do operations and $4 \%$ required circulatory arrest. Baseline demographics were comparable in both groups. Detailed preoperative characteristics of the patients are given in Table 3. There was no major difference in the use of antiplatelet and/ or anticoagulation therapy prior to surgery except for a significantly higher rate of coumadin therapy within the ROTEM ${ }^{\circ}$-group (Table 3 ).

Table 2 Transfusion protocol for the control group

\begin{tabular}{ll}
\hline Trigger: & drainage loss $>200 \mathrm{ml} / \mathrm{h}$ or $100 \mathrm{ml} / 30$ min at 30 min after \\
arrival at the ICU
\end{tabular}

Transfusion of RBCs according to the haemoglobin level of the blood-gasanalysis. Target value $>8.0 \mathrm{~g} / \mathrm{dl}$

${ }^{\mathrm{a}} \mathrm{FFP}(15 \mathrm{ml} / \mathrm{kg} \mathrm{KG}):<58 \mathrm{~kg}$ body weight - $>3 \mathrm{FFP}, 58-75 \mathrm{~kg}$ body weight $->4$ FFP, 75-92 kg body weight - $>5$ FFP, $>92 \mathrm{~kg}$ body weight $->6$ FFP

The entries in boldface represent the administered blood-components
Table 3 Preoperative baseline characteristics of all 104 patients included in the study

\begin{tabular}{|c|c|c|c|c|}
\hline Variables & OVERALL & CONTROL & ROTEM $^{\circledast}$ & $p$-value \\
\hline \multicolumn{5}{|l|}{ Baseline values } \\
\hline Number, n (\%) & 104 & $52(50)$ & $52(50)$ & \\
\hline Age [years] & $\begin{array}{l}67.2 \pm \\
10.4\end{array}$ & $68.1 \pm 9.9$ & $\begin{array}{l}66.4 \pm \\
12.9\end{array}$ & 0.62 \\
\hline Male sex, n (\%) & $84(80.8)$ & $45(87)$ & $39(75)$ & 0.21 \\
\hline BMl & $\begin{array}{l}26[24.0 ; \\
29.0]\end{array}$ & 25 [23.3;28.8] & $\begin{array}{l}27[24.3 ; \\
30.0]\end{array}$ & 0.11 \\
\hline Log. EuroSCORE [\%] & $7.0 \pm 8.8$ & $9.6 \pm 9.8$ & $8.2 \pm 7.5$ & 0.72 \\
\hline STS - Score [\%] & $2.4 \pm 2.7$ & $3.1 \pm 3.1$ & $3.3 \pm 3.2$ & 0.84 \\
\hline Biplane LVEF [\%] & $\begin{array}{l}60.0 \\
{[45.0 ;} \\
65.0]\end{array}$ & $59.5[45.0 ; 65.0]$ & $\begin{array}{l}60.0 \\
{[50.0 ;} \\
65.0]\end{array}$ & 0.45 \\
\hline $\begin{array}{l}\text { Diabetes mellitus, } \mathrm{n} \\
(\%)\end{array}$ & $33(31.7)$ & $16(31)$ & $17(33)$ & 1.00 \\
\hline COPD, n (\%) & $7(6.7)$ & $2(4)$ & $5(10)$ & 0.44 \\
\hline Creatinine $[\mathrm{mmol} / \mathrm{l}]$ & $\begin{array}{l}91[81.0 ; \\
115.0]\end{array}$ & $92.5[80.0 ; 109.0]$ & $\begin{array}{l}101 \\
{[82.5 ;} \\
119.0]\end{array}$ & 0.11 \\
\hline Re-operation, n (\%) & $14(13.5)$ & $7(14)$ & $7(14)$ & 1.00 \\
\hline $\begin{array}{l}\text { Deep hypothermic } \\
\text { circulatory arrest, } n \\
\text { (\%) }\end{array}$ & $4(3.8)$ & $2(4)$ & $2(4)$ & 1.00 \\
\hline $\begin{array}{l}\text { Aortic valve surgery, } \mathrm{n} \\
(\%)\end{array}$ & $50(48.0)$ & $23(44.2)$ & $27(51.9)$ & 0.74 \\
\hline CABG, n (\%) & $28(26.9)$ & $16(30.7)$ & $12(23.1)$ & 0.41 \\
\hline $\begin{array}{l}\text { Combined surgery }{ }^{a}, \mathrm{n} \\
(\%)\end{array}$ & $26(25.0)$ & $13(25.0)$ & $13(25.0)$ & 1.00 \\
\hline Acetylsalic acid, n (\%) & $62(58)$ & $33(64)$ & $29(56)$ & 0.54 \\
\hline Clopidogrel, n (\%) & $10(9)$ & $4(8)$ & $6(12)$ & 0.52 \\
\hline Coumadine, n (\%) & $8(8)$ & $1(2)$ & $7(14)$ & 0.03 \\
\hline LM Heparin, n (\%) & $21(20)$ & $9(17)$ & $12(23)$ & 0.47 \\
\hline Heparin, n (\%) & $10(9)$ & $3(6)$ & $7(14)$ & 0.20 \\
\hline
\end{tabular}

$B M I$ body mass index, LVEF left ventricular ejection fraction, COPD chronic obstructive pulmonary disease, $L M$ Heparin low-molecular subcutaneous heparin. ${ }^{\text {a }}$ : Aortic valve surgery in combination with CABG. Data presented as numbers (\%), mean \pm SD or as median (interquartile range)

As shown in Table 4, perioperative values were not statistically different in both groups except for a higher heparin dose in the ROTEM $^{\circ}$-group. Intraoperative transfusion requirements (prior to randomization) regarding red blood cells, platelets, plasma, fibrinogen, pooled factors or antifibrinolytic agents were comparable. At the time of the interim analysis, $75 \%$ more of the patients initially estimated were included in the study while the primary endpoint in the use of allogenic blood products could not be reached (Table 4).

In the postoperative course (after randomization), the ROTEM $^{-}$-based algorithm was associated with significantly less aprotinin, but increased fibrinogen usage. Requirements for RBCs, FFPs, platelets, PPSB, AT3 and 
Table 4 Clinical data of both randomized groups

\begin{tabular}{|c|c|c|c|}
\hline Variables & CONTROL & ROTEM $^{\oplus}$ & $p$-value \\
\hline \multicolumn{4}{|l|}{ Perioperative details } \\
\hline Number, n (\%) & $52(50)$ & $52(50)$ & \\
\hline CPB time [min.] & $109.2 \pm 74.6$ & $119.8 \pm 60.6$ & 0.44 \\
\hline Cross-clamp time [min] & $71.5 \pm 51.0$ & $77.3 \pm 39.5$ & 0.33 \\
\hline Heparine dosage [IU] & $\begin{array}{l}28,249.0 \pm 10 \\
419.0\end{array}$ & $\begin{array}{l}33,364.6 \pm 12 \\
000.3\end{array}$ & 0.01 \\
\hline Protamine dosage [IU] & $\begin{array}{l}28,580.0 \pm \\
9165.4\end{array}$ & $\begin{array}{l}28,851.1 \pm \\
8257.4\end{array}$ & 0.47 \\
\hline RBCs [units] & $1.1 \pm 1.7$ & $1.0 \pm 1.9$ & 0.48 \\
\hline FFPs [units] & $0.9 \pm 1.7$ & $0.5 \pm 1.1$ & 0.38 \\
\hline Platelet concentrates [units] & $0.1 \pm 0.5$ & $0.2 \pm 0.5$ & 0.97 \\
\hline Fibrinogen [g] & $0.1 \pm 0.6$ & $0.1 \pm 0.4$ & 0.71 \\
\hline PPSB [IU] & $137.3 \pm 566.4$ & $135.4 \pm 422.0$ & 0.50 \\
\hline AT3 [IU] & $137.3 \pm 566.4$ & $83.3 \pm 331.6$ & 0.72 \\
\hline Aprotinin, [Mio IU] & $0.3 \pm 0.7$ & $1.1 \pm 7.2$ & 0.09 \\
\hline Tranexamic acid [g] & $1.6 \pm 1.5$ & $1.9 \pm 1.5$ & 0.22 \\
\hline $\begin{array}{l}\text { ACT (prior to ICU transfer) } \\
\text { [sec] }\end{array}$ & $135.4 \pm 16.5$ & $132.3 \pm 20.3$ & 0.09 \\
\hline Hct (prior to ICU transfer) [\%] & $28.9 \pm 3.9$ & $28.6 \pm 4.1$ & 0.70 \\
\hline $\begin{array}{l}\text { Temperature (prior to ICU } \\
\text { transfer) }\left[{ }^{\circ} \mathrm{C}\right]\end{array}$ & $36.3[36.0 ; 36.6]$ & $36.4[35.9 ; 36.6]$ & 0.78 \\
\hline \multicolumn{4}{|l|}{ Postoperative - ICU } \\
\hline RBCs [units] & $5.2+8.1$ & $4.4+3.7$ & 0.73 \\
\hline FFPs [units] & $3.2 \pm 4.7$ & $2.2 \pm 4.1$ & 0.17 \\
\hline Platelet concentrates [units] & $0.7 \pm 1.1$ & $0.4 \pm 0.8$ & 0.16 \\
\hline Fibrinogen [g] & $0.2 \pm 0.6$ & $0.8 \pm 1.1$ & 0.01 \\
\hline PPSB [IU] & $58.8 \pm 420.1$ & $81.6 \pm 399.8$ & 0.61 \\
\hline AT3 [IU] & $19.6 \pm 140.0$ & $20.4 \pm 142.9$ & 1.00 \\
\hline Aprotinin [Mio IU] & $0.5 \pm 0.9$ & $0.1 \pm 0.4$ & 0.01 \\
\hline Tranexamic acid [g] & $0.1 \pm 0.4$ & $0.0 \pm 0.3$ & 1.00 \\
\hline $\begin{array}{l}\text { Blood loss within first } 24 \mathrm{~h} \\
{[\mathrm{ml}]}\end{array}$ & $1867.4 \pm 827.4$ & $1599.1 \pm 834.3$ & 0.07 \\
\hline Re-thoracotomy, n (\%) & $15(29)$ & $13(25)$ & 0.83 \\
\hline 30-day mortality, n (\%) & $4(8)$ & $1(2)$ & 0.17 \\
\hline 5-year mortality, n (\%) & $6(12)$ & $2(4)$ & 0.14 \\
\hline
\end{tabular}

Data presented as numbers (\%), mean \pm SD or as median (interquartile range)

tranexamic acid were comparable. Overall, there was a strong trend towards less bleeding within the first $24 \mathrm{~h}$ in the ROTEM ${ }^{\circ}$ group visible however not reaching statistical significance $(p=0.066)$. Rate of re-thoracotomy for bleeding was comparable in both groups. A "surgical" bleeding could be identified in $21 \%$ (11 out of 15 ) in the control and in $17 \%$ (9 out of 13 ) in the ROTEM $^{\circ}$ group $(p=0.8)$.

Within 30 days, one patient in the $\operatorname{ROTEM}^{\circ}(2 \%)$ and 3 patients in the control group (6\%) suffered a stroke $(p=0.618)$. Requirement for dialysis due to acute renal failure was $6 \%(n=3)$ in the control and $12 \%(n=6)$ in the $\operatorname{ROTEM}^{\circ}$ group $(p=0.488)$. Ventilation times were prolonged but comparable in both groups (control: $95.9 \pm 197.2$ vs. $\left.\operatorname{ROTEM}^{\circ}: 97.7 \pm 159.3 \mathrm{~h} ; p=0.17\right)$.

Thirty-day mortality rate was $8 \%(n=4)$ in the control and $2 \%(n=1)$ in the ROTEM $^{\circ}$ group $(p=0.17)$. The patient in the $\mathrm{ROTEM}^{\circ}$ group died on postoperative day (POD) 12 due to multi-organ failure. In the control group, one patient died due to acute myocardial infarction (POD 3), one due to right heart failure (POD 29), one due to unclear reasons (POD 15) and one due to multi-organ failure (POD 27). At 5-year follow-up mortality rate was $12 \%(n=6)$ in the control and $4 \%(n=2)$ in the $\operatorname{ROTEM}^{\circ}$ group $(p=0.14)$.

\section{Patients at "high-risk" for bleeding (long CPB times)}

Out of the total 104 patients 55 patients were identified that underwent procedures with a long CPB-time known to increase the risk for diffuse coagulopathy. As shown in Table 5, baseline and perioperative values were not statistically different in both treatment groups and intraoperative transfusion requirements again were comparable although $\mathrm{ROTEM}^{\circ}$ patients received more aprotinin intraoperatively.

After randomization (postoperative course), the two different treatment protocols resulted in significantly different distribution of coagulation products requirements. Whereas ROTEM $^{\circ}$ patients received significantly less platelets concentrates and aprotinin, control patients required less fibrinogen. Re-thoracotomy rate was comparable and 3 patients in the control versus none in the ROTEM $^{\circ}$ group died. At 5 -year follow-up there was a significant improved survival in the ROTEM $^{\circ}$ group $(0 \%$ vs. $15 \% ; p=0.03)$. Overall, ROTEM $^{\circ}$ patients suffered significantly less blood loss within the first $24 \mathrm{~h}$ (ROTEM ${ }^{\ominus}$ : $1538.2 \pm 806.4 \mathrm{ml}$ vs. control: $2056.8 \pm 974.5$ $\mathrm{ml} ; p=0.032$; Fig. 1).

\section{Cost analysis}

A cumulative cost analysis of all blood component products revealed cost savings of $128.50 €$ per patient $(-10 \%)$ treated in the "point-of-care" 4-chamber $\mathrm{ROTEM}^{\circ}$ group (Table 6). However, in the subgroup of patients with "long" CPB-times cost savings increased to even $619.75 €$ per patient (Table 7, Fig. 2). In total, average costs of all blood component products per patient decreased in this group from $1723.50 €$ to $1103.75 €(-36 \%)$. In addition, costs savings were not annihilated by the additional cost for ROTEM ${ }^{\bullet}$ testing (on average: $68.30 €$ per patient).

\section{Discussion}

Significant postoperative bleeding is a problem well known in any cardiac surgical center. It has to be expected in $5-10 \%$ of patients depending on the type of 
Table 5 Clinical characteristics of 55 patients with "long" CPB time $(\geq 115 \mathrm{~min})$

\begin{tabular}{|c|c|c|c|}
\hline Variables & CONTROL & ROTEM $^{\oplus}$ & $p$-value \\
\hline \multicolumn{4}{|l|}{ Baseline values } \\
\hline Number, n (\%) & $26(47)$ & $29(53)$ & \\
\hline Age [years] & $66.9 \pm 11.1$ & $66.7 \pm 10.0$ & 0.87 \\
\hline Male sex, n (\%) & $22(85)$ & $26(90)$ & 0.70 \\
\hline BMI & $\begin{array}{l}24.5[23.0 ; \\
29.0]\end{array}$ & $27.0[25.0 ; 29.5]$ & 0.07 \\
\hline Log. EuroSCORE [\%] & $10.2 \pm 10.5$ & $7.4 \pm 6.9$ & 0.40 \\
\hline STS - Score [\%] & $3.7 \pm 3.9$ & $3.3 \pm 3.1$ & 0.73 \\
\hline Biplane LVEF [\%] & $\begin{array}{l}60.0[41.5 ; \\
65.0]\end{array}$ & $59.0[50.0 ; 65.0]$ & 1.00 \\
\hline Diabetes mellitus, n (\%) & $7(27)$ & $7(24)$ & 1.00 \\
\hline COPD, n (\%) & $1(4)$ & $5(17)$ & 0.20 \\
\hline Creatinine $[\mathrm{mmol} / \mathrm{l}]$ & $\begin{array}{l}98.0[80.0 ; \\
109.3]\end{array}$ & $\begin{array}{l}106.0[86.5 \\
130.5]\end{array}$ & 0.07 \\
\hline $\begin{array}{l}\text { Deep hypothermic circulatory } \\
\text { arrest, } \mathrm{n}(\%)\end{array}$ & $2(8)$ & $1(3)$ & 0.60 \\
\hline Re-operation, n (\%) & $2(8)$ & $4(14)$ & 0.67 \\
\hline \multicolumn{4}{|l|}{ Perioperative details } \\
\hline CPB time $[\mathrm{min}]$ & $161.4 \pm 59.8$ & $159.3 \pm 41.3$ & 0.93 \\
\hline Cross-clamp time [min] & $105.8 \pm 41.9$ & $99.3 \pm 31.7$ & 0.84 \\
\hline Heparin [IU] & $\begin{array}{l}31,528.0 \pm \\
9526.2\end{array}$ & $\begin{array}{l}34,925.9 \pm 10 \\
049.6\end{array}$ & 0.16 \\
\hline Protamin [IU] & $\begin{array}{l}30,720.0 \pm \\
7924.2\end{array}$ & $\begin{array}{l}31,000.0 \pm \\
5699.1\end{array}$ & 0.46 \\
\hline RBCs [units] & $1.4 \pm 2.0$ & $1.2 \pm 2.1$ & 0.45 \\
\hline FFPs [units] & $1.2 \pm 1.9$ & $0.7 \pm 1.2$ & 0.52 \\
\hline Platelet concentrates [units] & $0.3 \pm 0.7$ & $0.1 \pm 0.4$ & 0.20 \\
\hline Fibrinogen $[\mathrm{g}]$ & $0.2 \pm 0.7$ & $0.1 \pm 0.5$ & 1.00 \\
\hline PPSB [IU] & $\begin{array}{l}200.0 \pm \\
707.1\end{array}$ & $166.7 \pm 500.0$ & 1.00 \\
\hline AT3, IU, mean $\pm S D$ & $\begin{array}{l}200.0 \pm \\
707.1\end{array}$ & $129.6 \pm 429.5$ & 1.00 \\
\hline Aprotinin [Mio IU] & $0.6 \pm 0.9$ & $1.9 \pm 9.6$ & 0.03 \\
\hline Tranexamic acid [g] & $1.6 \pm 1.5$ & $1.8 \pm 1.5$ & 1.00 \\
\hline ACT (prior to ICU transfer) [sec] & $134.1 \pm 16.1$ & $130.7 \pm 20.2$ & 0.18 \\
\hline Hct (prior to ICU transfer) [\%] & $28.1 \pm 4.4$ & $28.4 \pm 3.8$ & 0.71 \\
\hline $\begin{array}{l}\text { Temperature (prior to ICU } \\
\text { transfer) }\left[{ }^{\circ} \mathrm{C}\right]\end{array}$ & $\begin{array}{l}36.4[36.0 ; \\
36.6]\end{array}$ & $36.3[36.0 ; 36.6]$ & 0.70 \\
\hline \multicolumn{4}{|l|}{ Postoperative - ICU } \\
\hline RBCs [units] & $7.1+10.8$ & $3.8+3.8$ & 0.28 \\
\hline FFPs [units] & $4.2 \pm 5.6$ & $1.7 \pm 2.8$ & 0.07 \\
\hline Platelet concentrates [units] & $1.1 \pm 1.3$ & $0.4 \pm 0.6$ & 0.02 \\
\hline Fibrinogen $[\mathrm{g}]$ & $0.2 \pm 0.6$ & $0.9 \pm 1.2$ & 0.01 \\
\hline PPSB [IU] & $0.0 \pm 0.0$ & $0.0 \pm 0.0$ & 1.00 \\
\hline AT3 [IU] & $40.0 \pm 200.0$ & $0.0 \pm 0.0$ & 0.48 \\
\hline Aprotinin [Mio IU] & $0.4 \pm 0.8$ & $0.0 \pm 0.0$ & 0.02 \\
\hline
\end{tabular}

Table 5 Clinical characteristics of 55 patients with "long" CPB time ( $\geq 115 \mathrm{~min}$ ) (Continued)

\begin{tabular}{llll}
\hline Variables & CONTROL & ROTEM $^{\circledR}$ & $p$-value \\
\hline Tranexamic acid [g] & $0.1 \pm 0.4$ & $0.1 \pm 0.4$ & 1.00 \\
Blood loss within first 24 h [ml] & $\begin{array}{l}2056.8 \pm \\
974.5\end{array}$ & $1538.2 \pm 806.4$ & 0.03 \\
Re-thoracotomy, n (\%) & $7(27)$ & $6(21)$ & 0.75 \\
30-day mortality, n (\%) & $3(12)$ & $0(0)$ & 0.10 \\
5-year mortality, n (\%) & $4(15)$ & $0(0)$ & 0.03 \\
\hline
\end{tabular}

Data presented as numbers (\%), mean $\pm \mathrm{SD}$ or as median (interquartile range)

surgery and potential pre-existing risk factors [3]. It has been demonstrated that transfusion requirements have a considerable impact on postoperative outcome and are associated with increased mortality [17].

Optimally, treatment algorithms would be based on coagulation tests with rapid results, independent of heparin and capable of assessing the current function of the different coagulation system components. Theoretically, modified thrombelastometry systems designed as a "point-of-care" test seems well suited and have been proven to further reduce transfusion requirements in a small prospectively randomized trial compared to an algorithm solely based on standard tests [18].

An advanced "point-of-care" system (ROTEM ${ }^{\circ}$ ) has been introduced that allows for simultaneous testing in four chambers with different activation agents ("intrinsic" / "extrinsic"), platelets blockade and aprotinin or heparinase addition. The clinical introduction of this thrombelastometry-based blood component treatment has been demonstrated to reduce transfusion requirements and costs $[11,15,19,20]$. The aim was to study a

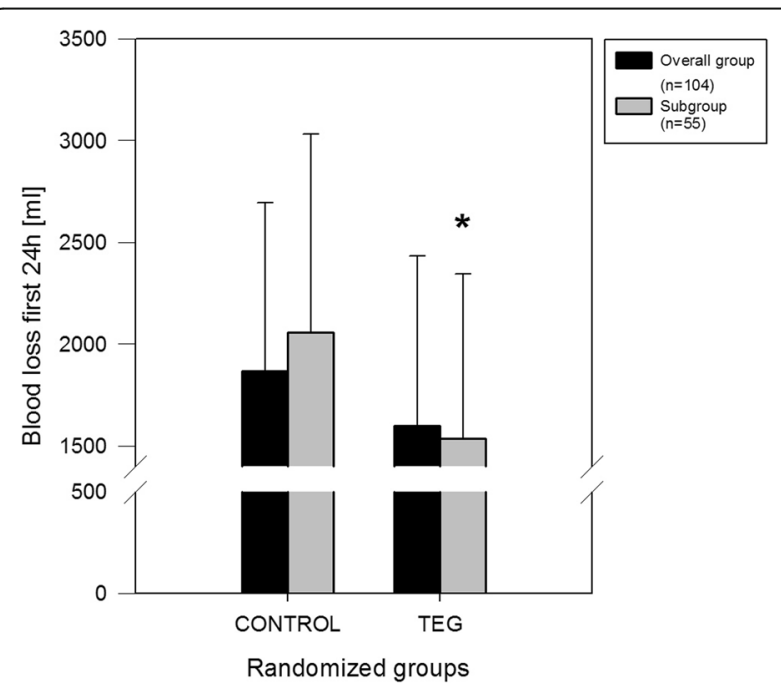

Fig. 1 * evidence for a significant difference between control group and ROTEM ${ }^{\oplus}$-guided therapy in the subgroup of patients with long CPB-times ( $\geq 115$ min.; $p=0.032$ ) 
Table 6 Cumulative cost analysis of all blood products ( $n=104$ patients)

\begin{tabular}{|c|c|c|}
\hline Variables & CONTROL & ROTEM $^{\odot}$ \\
\hline Number & $52(50)$ & $52(50)$ \\
\hline \multicolumn{3}{|l|}{ intraoperative } \\
\hline RBCs $(70 €)$ & 3780 & 3290 \\
\hline FFPs $(51 €)$ & 2346 & 1326 \\
\hline Platelet concentrates (unit: $500 €$ ) & 4000 & 3500 \\
\hline Fibrinogen, $1 \mathrm{~g}(287.5 €)$ & 2013 & 1725 \\
\hline PPSB, 500 IU (120 €) & 1680 & 1560 \\
\hline AT3, $1000 \mathrm{IU}(70 €)$ & 490 & 280 \\
\hline Aprotinin (2.5 Mio IU $123.75 €$ ) & 668 & 2574 \\
\hline Tranexamic acid (500 mg: $7.63 €$ ) & 1206 & 1373 \\
\hline Cumulative costs & 16,183 & 15,628 \\
\hline \multicolumn{3}{|l|}{ ICU (after randomization) } \\
\hline RBCs $(70 €)$ & 18,690 & 14,980 \\
\hline FFPs (51 €) & 8313 & 5559 \\
\hline Platelet concentrates (unit: $500 €$ ) & 17,500 & 10,000 \\
\hline Fibrinogen, $1 \mathrm{~g}(287.5 €)$ & 2875 & 11,500 \\
\hline PPSB, 500 IU (120 €) & 720 & 960 \\
\hline AT3, $1000 \mathrm{IU}(70 €)$ & 70 & 70 \\
\hline Aprotinin (2.5 Mio IU $123.75 €$ ) & 1188 & 198 \\
\hline Tranexamic acid (500 mg: $7.63 €$ ) & 61 & 31 \\
\hline Cumulative costs & 49,417 & 43,298 \\
\hline Overall cumulative costs $[€]$ & 65,600 & 58,926 \\
\hline Costs per patient [€] & 1261.5 & 1133 \\
\hline Costs savings per patient $[€]$ & - & 128.5 \\
\hline
\end{tabular}

thrombelastometry-based algorithm in comparison to a protocol based on classic coagulation tests known to be superior to "empiric" treatment.

Overall we observed comparable transfusion requirements between the ROTEM $^{\circledR}$-guided and the classic coagulation test guided group regarding RBCs, FFPs, platelets and pooled factors (PPSB). Our ROTEM ${ }^{\oplus}$-guided blood component treatment algorithm was at least as effective and safe as protocols based on classic coagulation tests. However, distribution blood products differed between both groups. ROTEM ${ }^{\triangleright}$ patients received more fibrinogen but significantly less aprotinin, as already stated in a recent study [11].

There was a clear trend towards less 24 h-bleeding visible in the ROTEM $^{\ominus}$ group, also more patients were on coumadin treatment preoperatively and heparin dosages were significantly higher in the ROTEM $^{\odot}$ group. When analyzing the subgroup of "high-risk" patients with long CPB-times known to be prone to diffuse coagulopathy ROTEM $^{\circledR}$ guided treatment resulted in significantly less 24 h-drainage loss and an improved 5-year survival. It seems that the more specific approach of the ROTEM ${ }^{\circ}$
Table 7 Cumulative cost analysis of all blood products ( $n=55$ patients with (PB time $\geq 115 \mathrm{~min}$ )

\begin{tabular}{|c|c|c|}
\hline Variables & CONTROL & ROTEM $^{\circledast}$ \\
\hline Number & $26(47 \%)$ & $29(53 \%)$ \\
\hline \multicolumn{3}{|l|}{ intraoperative } \\
\hline RBCs (unit: $70 €$ ) & 2520 & 2240 \\
\hline FFPs (unit: $51 €$ ) & 1530 & 1020 \\
\hline Platelet concentrates (unit: $500 €$ ) & 4000 & 1500 \\
\hline Fibrinogen (1 g: $287.5 €$ ) & 1438 & 1150 \\
\hline PPSB (500 IU: $120 €$ ) & 1200 & 1080 \\
\hline AT3 (1000 IU: $70 €$ ) & 350 & 245 \\
\hline Aprotinin (2.5 Mio IU $123.75 €$ ) & 668 & 2574 \\
\hline Tranexamic acid (500 mg: $7.63 €$ ) & 595 & 732 \\
\hline Cumulative costs [€] & 12,301 & 10,541 \\
\hline \multicolumn{3}{|l|}{ ICU (after randomization) } \\
\hline RBCs (unit: $70 €$ ) & 12,460 & 7140 \\
\hline FFPs (unit: $51 €$ ) & 5304 & 2397 \\
\hline Platelet concentrates (unit: $500 €$ ) & 13,000 & 5000 \\
\hline Fibrinogen (1 g: $287.5 €$ ) & 1150 & 6900 \\
\hline PPSB (500 IU: $120 €$ ) & 0 & 0 \\
\hline AT3 (1000 IU: $70 €$ ) & 70 & 0 \\
\hline Aprotinin (2.5 Mio IU: $123.75 €)$ & 495 & 0 \\
\hline Tranexamic acid (500 mg: $7.63 €$ ) & 31 & 31 \\
\hline Cumulative costs [€] & 32,510 & 21,468 \\
\hline Overall cumulative costs $[€]$ & 44,811 & 32,009 \\
\hline Costs per patient [€] & 1723.5 & 1103.8 \\
\hline Costs savings per patient $[€]$ & - & 619.8 \\
\hline
\end{tabular}

based algorithm resulted in less bleeding. In addition, the ability to deliver the specific treatment faster than with standard tests may have contributed to the observed benefit. The ROTEM $^{\odot}$ protocol requires $10 \mathrm{~min}$ until the results allow for a decision if additional protamine, FFPs or platelets are required and after $30 \mathrm{~min}$ measurements will guide platelets, fibrinogen or antifibrinolytic agent therapy. In contrast, at least in our center, results of standard coagulation test are rarely available in less than one hour.

Rate of re-thoracotomy was not different between the two groups. To differentiate between surgical bleeding and diffuse coagulopathy is always a difficult task. Standard coagulation tests have been shown to be of no help at all in this scenario: negative predictive accuracy has been reported with $50 \%$ - basically the same as guessing [5]. In contrast, $\mathrm{ROTEM}^{\bullet}$ is more accurate and allows for a negative predictive value (excluding diffuse coagulopathy) of $82 \%$, however positive prediction is less [5, 21]. In conclusion, ROTEM $^{\circledR}$ might be helpful in the assessment of surgical versus diffuse bleeding but overall accuracy seems to be not sufficient. Thus, the decision 


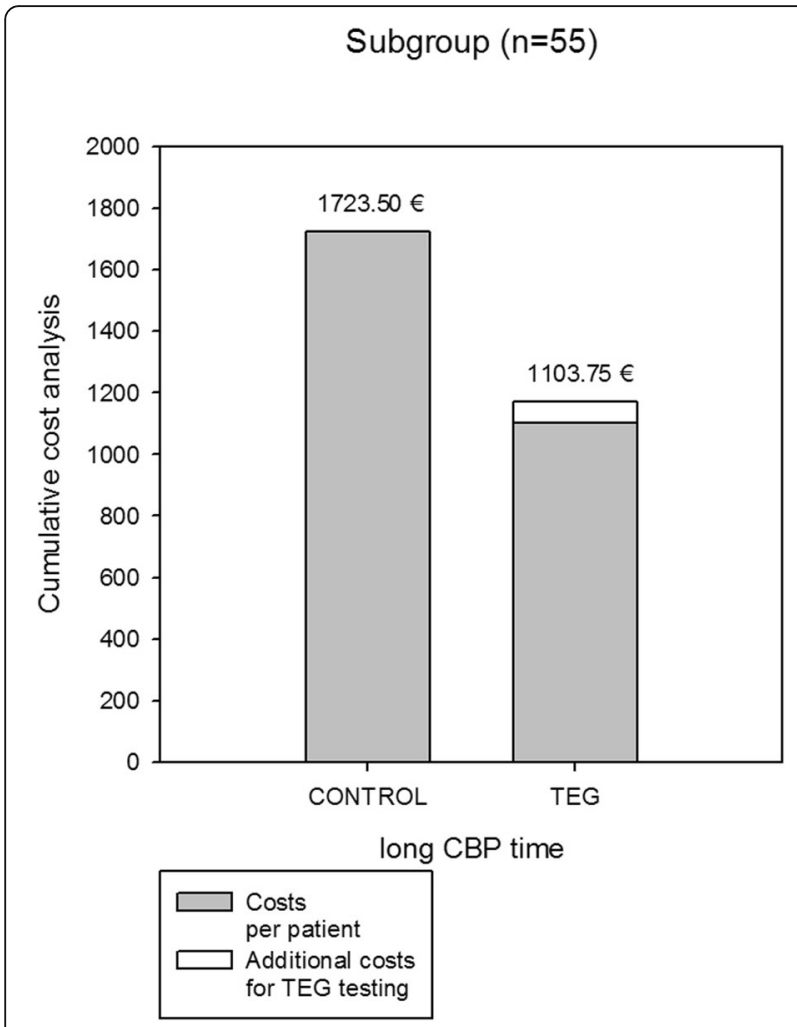

Fig. 2 A cumulative cost analysis of all blood component products in the subgroup of patients with "long" CPB-times revealed cost savings of $619.8 €$ per patient treated in the "point-of-care" 4chamber ROTEM ${ }^{\oplus}$. Costs savings were not annihilated by the additional costs for ROTEM ${ }^{\circledast}$ testing (on average: $68.3 €$ per patient)

for re-thoracotomy in our series was based on clinical judgment and only partially based on ROTEM ${ }^{\circ}$ or standard coagulation test. As in only $21 \%$ of control and in $17 \%$ of ROTEM $^{\circ}$ patients a surgical bleeding could be identified, a substantial number of bleeding events could have been avoided with a better decision making protocol. Ideally, specific predictive values derived from ROTEM $^{\circ}$ or other coagulation tests might become available in the future facilitating a more evidence based approach if or if not to perform a rethoracotomy [22, 23].

Avarage costs for the blood products used in this study were estimated according to Spalding et al. [11]. Consistent with previous studies we could confirm in our prospective randomized trial a cost-reduction when using a specific ROTEM ${ }^{\circ}$ based treatment algorithm (Tables 6 and 7). Cost-savings were not counterbalanced by the additional costs for thrombelastometry testing (Fig. 2). Thus, it has to be considered that the implementation of such a ROTEM ${ }^{\circ}$-based protocol requires substantial human training and a dedicated and highly motivated ICU team as the work-load is increased due to the requirements to manually perform the $\mathrm{ROTEM}^{\circ}$ tests on the
ICU in comparison to simply sending a blood sample to the laboratory for traditional coagulation tests.

Another issue of concern is the accuracy and reproducibility of a "point-of-care" test performed manually by "non-specialized" physicians outside the laboratory environment. However, for the ROTEM $^{\circ}$ device sufficient reproducibility and stability of measurements have been reported [24-26]. The use of fully-automated systems like ROTEM $^{\circ}$ sigma need to be disseminated more widely to stimulate a broader use of this beneficial technology.

We observed a beneficial effect of the ROTEM ${ }^{\circ}$ algorithm predominantly in the subgroup of "high-risk" patients with prolonged CPB-times. However, we have to consider that due to the study design $\mathrm{ROTEM}^{\circ}$ was compared to a well-structured and evidence based standard transfusion protocol.

In this study, there was an overall trend towards improved survival at 30-days, however in the subgroup of "high-risk" patients known to be prone to diffuse coagulopathy a significant reduction in 5-year mortality was proven in the long-term follow-up. Standard treatment algorithms have been proven to significantly reduce transfusion requirements in comparison with "empiric" therapy $[1,5,7,27]$. Hence, the observed benefit of a ROTEM $^{\circ}$-guided protocol has been shown to be effective and safe in a prospective randomized trial in cardiac surgical patients that suffer postoperative bleeding and secondly a further reduction of bleeding, costs and mortality could be demonstrated. In our opinion, "empiric" blood component therapy should not be used in clinical practice. For regular patients both, standard and ROTEM $^{\ominus}$-based specific treatment algorithms have been shown repeatedly superior providing a true clinical benefit for the patients $[1,5]$.

With regard to a recent updated meta-analysis by Serraino and collegues [28] ROTEM-guided algorithms lead to a significant reduction in transfusion of RBCs, FFPs, platelets and the rate of severe acute kidney injury compared to CONTROL groups. There was also an improvement seen in mortality, number of reoperations for bleeding, ventilation times, shorter ICU length of stay and hospital stay, but none of them significantly reduced. The authors concluded that viscoelastic testing lacks clinical effectiveness with only weak evidence and low predictive accuracy for coagulopathic bleeding. However, a treatment algorithm based on "point-of-care" 4-chamber ROTEM $^{\circ}$ seemed to be at least as effective as standard therapy with improvement in a broad range of relevant clinical parameters. Furthermore, our incidence of postoperative acute kidney injury was rather low for a sample of patients with significant bleeding. Interestingly, our results are supported by newer studies, who demonstrated that a low nadir hematocrit (cutoff value of about 24\%) was inversely associated with acute kidney injury [29-31]. 
A ROTEM ${ }^{\circ}$ guided protocol seems to be capable to further reduce bleeding, costs and mortality as it allows for a highly specific and fast therapy tailored to the functional coagulation status of the individual patient and might be especially beneficial in "high-risk" patients prone to bleeding complications [14].

\section{Limitations}

The major limitation of this trial is that patients with aortic valve replacement as well as with combined CABG were included. Thus, our data might include a considerable preoperative inhomogeneity regarding the incidence of von-Willebrand-Syndrom. In addition, limited evidence is available in regard to the structure of the ROTEM $^{\circ}$ algorithm and baseline reference values [32] which might not allow comparability between "regular" and cardiac surgical patients after CPB. Furthermore, the small number of patients as well as surgical bleeding, that cannot be treated based on thromboelastometry, may have biased our results. However, it is still a unique randomized trial assessing ROTEM $^{\circ}$ effectiveness in cardiac surgical patients in case of postoperative bleeding. Furthermore, the rate of inadequate platelet response was not assessed in our study, but might be equally distributed within the groups due to randomization.

Within the duration of the trial Trasylol had been withdrawn from the marked. Subsequently, aprotinin was replaced by tranexamic acid. However, this change in treatment affected both groups equally and in addition tranexamic acid has been shown to be as effective as aprotinin in several trials [33].

\section{Conclusion}

Significant postoperative bleeding after cardiac surgical procedures is a well-known problem with dramatic impact on clinical outcome, mortality and costs. Based on the results of our randomized trial comparing a "4chamber" modified thromboelastometry-guided protocol with an algorithm based on standard coagulation testing the $\mathrm{ROTEM}^{\circ}$ approach proved to be at least as effective and safe as the standard approach. In patients with long CPB-times prone for diffuse coagulopathy ROTEM ${ }^{\circ}$ guided therapy may result in less bleeding, a marked reduction in costs and long-term mortality.

\section{Supplementary information}

Supplementary information accompanies this paper at https://doi.org/10. 1186/s12871-019-0875-7.

Additional file 1. CONSORT 2010 Checklist, CONSORT 2010 checklist of information to include when reporting a randomised trial.

Additional file 2. CONSORT 2010 Flow Diagram, Flow diagram of the progress through the phases of the prospective randomised trial of the ROTEM $^{\oplus}$ and CONTROL group.

\section{Abbreviations}

ACT: activated clotting time (last value in the operating theatre);

APC: activated protein C; APTEM: rotational thromboelastometry that shows hyperfibrinolysis by inhibition with aprotinin; AT3: anti-thrombin 3;

AVR: aortic valve replacement; CABG: coronary artery bypass graft;

CONSORT: Consolidated Standards of Reporting Trials; COPD: chronic

obstructive pulmonary disease; CPB: cardiopulmonary bypass; CT: clotting time; dl: deziliter; EuroSCORE: European System for Cardiac Operative Risk Evaluation; FFPs: fresh frozen plasma; FIBTEM: rotational thromboelastometry that shows isolated fibrinogen contribution; Hct: hematocrit; HEPTEM: rotational thromboelastometry that shows heparin effects by inhibition with heparinase; HIT: heparine induced thrombocytopenia; ICU: intensive care unit; INR: international normalized ratio; INTEM: rotational thromboelastometry that shows intrinsic thromboelastometry;

IU: international units; kg: kilogramm; LVEF: left ventricular ejection fraction; MCF: maxium clot firmness; min: minute; $\mathrm{ml}$ : mililiter; $\mathrm{mm}$ : milimeter; $\mathrm{N}$ : number; POD: postoperative day; PPSB: prothrombin complex concentrate: pooled factors (Prothrombin-Proconvertin-Stuart FactorAntihemophilic Factor B); PTT: partial thromboplastin time; RBCs: packed red blood cells; ROTEM: rotational thromboelastometry; Sec: second; STS: Society of Thoracic Surgeons Score

\section{Acknowledgements}

Preliminary data were presented at the 25th Annual Meeting of the European Association for Cardio-Thoracic Surgery: https://academic.oup.com/ icvts/issue/13/Supplement_2.

\section{Authors' contributions}

JK, PMK and EG have drafted the trial and made substantial contributions to the design of the study. $\mathrm{MH}$ and JK analyzed and interpreted the patient data and were major contributors in writing the manuscript. MAB and SL have substantively revised it. All authors read and approved the final manuscript. All authors have agreed both to be personally accountable for the author's own contributions and to ensure that questions related to the accuracy or integrity of any part of the work, even ones in which the author was not personally involved, are appropriately investigated, resolved, and the resolution documented in the literature.

Funding

Not applicable.

\section{Availability of data and materials}

The trial protocol, datasets used and/or analysed during the current study are available from the corresponding author on reasonable request.

Ethics approval and consent to participate

After approval by the local ethics committee of the University of Leipzig, we herein confirm written consent obtained from each patient in accordance with the Declaration of Helsinki in order to report and publish the individual patient data obtained. A statement on consent to participate from each patient in the current study was obtained.

Consent for publication

Not applicable.

\section{Competing interests}

The authors declare that they have no competing interests.

\section{Author details}

${ }^{1}$ Department of Vascular Surgery, Cardiovascular Center, University of Leipzig, Liebigstr 20, 04103 Leipzig, Germany. ${ }^{2}$ Department of Cardiothoracic and Vascular Surgery, German Heart Center Berlin, Berlin, Germany. ${ }^{3}$ Department of Cardiac and Cardiovascular Surgery, University Heart Center Hamburg, Hamburg, Germany. ${ }^{4}$ Clinic of Cardiac Surgery, Heart Center, University of Leipzig, Leipzig, Germany. 
Received: 17 April 2019 Accepted: 24 October 2019

Published online: 06 November 2019

\section{References}

1. Deppe AC, Weber C, Zimmermann J, Kuhn EW, Slottosch I, Liakopoulos OJ, et al. Point-of-care thromboelastography/thromboelastometry-based coagulation management in cardiac surgery: a meta-analysis of 8332 patients. J Surg Res. 2016;203:424-33.

2. Sharma S, Kumar S, Tewari P, Pande S, Murari M. Utility of thromboelastography versus routine coagulation tests for assessment of hypocoagulable state in patients undergoing cardiac bypass surgery. Ann Card Anaesth. 2018;21:151-7.

3. Whitlock $\mathrm{R}$, Crowther MA, Ng HJ. Bleeding in cardiac surgery: its prevention and treatment--an evidence-based review. Crit Care Clin. 2005;21:589-610.

4. Karkouti K, Wijeysundera DN, Yau TM, Beattie WS, Abdelnaem E, McCluskey SA, et al. The independent association of massive blood loss with mortality in cardiac surgery. Transfusion. 2004;44:1453-62.

5. Wikkelso A, Wetterslev J, Moller AM, Afshari A. Thromboelastography (TEG) or thromboelastometry (ROTEM) to monitor haemostatic treatment versus usual care in adults or children with bleeding. Cochrane Database Syst Rev 2016:CD007871

6. Emmert MY, Salzberg SP, Theusinger OM, Felix C, Plass A, Hoerstrup SP, et al. How good patient blood management leads to excellent outcomes in Jehovah's witness patients undergoing cardiac surgery. Interact Cardiovasc Thorac Surg. 2011;12:183-8.

7. Avidan MS, Alcock EL, Da Fonseca J, Ponte J, Desai JB, Despotis GJ, et al. Comparison of structured use of routine laboratory tests or near-patient assessment with clinical judgement in the management of bleeding after cardiac surgery. Br J Anaesth. 2004;92:178-86.

8. Royston D, von Kier S. Reduced haemostatic factor transfusion using heparinase-modified thrombelastography during cardiopulmonary bypass. Br J Anaesth. 2001;86:575-8.

9. Theusinger OM, Spahn DR, Ganter MT. Transfusion in trauma: why and how should we change our current practice? Curr Opin Anaesthesiol. 2009;22:305-12.

10. Anderson L, Quasim I, Soutar R, Steven M, Macfie A, Korte W. An audit of red cell and blood product use after the institution of thromboelastometry in a cardiac intensive care unit. Transfus Med. 2006:16:31-9.

11. Spalding GJ, Hartrumpf M, Sierig T, Oesberg N, Kirschke CG, Albes JM. Cost reduction of perioperative coagulation management in cardiac surgery: value of "bedside" thrombelastography (ROTEM). Eur J Cardiothorac Surg. 2007;31:1052-7.

12. Lei Q, Chen L, Zhang Y, Fang N, Cheng W, Li L. Predictors of prolonged mechanical ventilation after aortic arch surgery with deep hypothermic circulatory arrest plus antegrade selective cerebral perfusion. J Cardiothorac Vasc Anesth. 2009;23:495-500.

13. Salis S, Mazzanti W, Merli G, Salvi L, Tedesco CC, Veglia F, et al. Cardiopulmonary bypass duration is an independent predictor of morbidity and mortality after cardiac surgery. J Cardiothorac Vasc Anesth. 2008;22:814-22.

14. Whiting D, DiNardo JA. TEG and ROTEM: technology and clinical applications. Am J Hematol. 2014;89:228-32.

15. Girdauskas E, Kempfert J, Kuntze T, Borger MA, Enders J, Fassl J, et al. Thromboelastometrically guided transfusion protocol during aortic surgery with circulatory arrest: a prospective, randomized trial. J Thorac Cardiovasc Surg. 2010;140:1117-24 e2.

16. Schulz KF, Altman DG, Moher D, Group C. CONSORT 2010 statement: updated guidelines for reporting parallel group randomised trials. BMC Med. 2010;8:18.

17. Vivacqua A, Koch CG, Yousuf AM, Nowicki ER, Houghtaling PL, Blackstone EH, et al. Morbidity of bleeding after cardiac surgery: is it blood transfusion, reoperation for bleeding, or both? Ann Thorac Surg. 2011;91:1780-90.

18. Shore-Lesserson L, Manspeizer HE, DePerio M, Francis S, Vela-Cantos F, Ergin MA. Thromboelastography-guided transfusion algorithm reduces transfusions in complex cardiac surgery. Anesth Analg. 1999;88:312-9.

19. Kultufan Turan S AB, Ayik I, Yagar S, Kazanci D, Karadeniz U. The role of rotational thromboelastgraphy on decision of blood transfusion in open heart surgery. . 2006:154-9.

20. Weber CF, Gorlinger K, Meininger D, Herrmann E, Bingold T, Moritz A, et al. Point-of-care testing: a prospective, randomized clinical trial of efficacy in coagulopathic cardiac surgery patients. Anesthesiology. 2012;117:531-47.

21. Cammerer U, Dietrich W, Rampf T, Braun SL, Richter JA. The predictive value of modified computerized thromboelastography and platelet function analysis for postoperative blood loss in routine cardiac surgery. Anesth Analg. 2003:96:51-7 table of contents.

22. Fleming K, Redfern RE, March RL, Bobulski N, Kuehne M, Chen JT, et al. TEGdirected transfusion in complex cardiac surgery: impact on blood product usage. J Extra Corpor Technol. 2017;49:283-90.

23. Spahn DR. TEG(R)- or ROTEM(R)-based individualized goal-directed coagulation algorithms: don't wait--act now! Crit Care. 2014;18:637.

24. Theusinger OM, Nurnberg J, Asmis LM, Seifert B, Spahn DR. Rotation thromboelastometry (ROTEM) stability and reproducibility over time. Eur J Cardiothorac Surg. 2010;37:677-83.

25. Drumheller BC, Stein DM, Moore LJ, Rizoli SB, Cohen MJ. Thromboelastography and rotational Thromboelastometry for the surgical Intensivist: a narrative review. J Trauma Acute Care Surg. 2019.

26. Hernandez Conte A, Perotti D, Farac L. Thromboelastrography (TEG) is still relevant in the 21st century as a point-of-care test for monitoring coagulation status in the cardiac surgical suite. Semin Cardiothorac Vasc Anesth. 2017:21:212-6.

27. Ranucci M, Baryshnikova E, Pistuddi V, Menicanti L, Frigiola A, Surgical et al. The effectiveness of 10 years of interventions to control postoperative bleeding in adult cardiac surgery. Interact Cardiovasc Thorac Surg 2017;24: 196-202.

28. Serraino GF, Murphy GJ. Routine use of viscoelastic blood tests for diagnosis and treatment of coagulopathic bleeding in cardiac surgery: updated systematic review and meta-analysis. Br J Anaesth. 2017;118:823-33.

29. Brescia AA, Wu X, Paone G, Heung M, Paugh TA, Shann KG et al. Effect of sex on nadir hematocrit and rates of acute kidney injury in coronary artery bypass. The Journal of thoracic and cardiovascular surgery 2019.

30. Crosina J, Lerner J, Ho J, Tangri N, Komenda P, Hiebert B, et al. Improving the prediction of cardiac surgery-associated acute kidney injury. Kidney international reports. 2017;2:172-9.

31. Ranucci M, Aloisio T, Carboni G, Ballotta A, Pistuddi V, Menicanti L, et al. Acute kidney injury and Hemodilution during cardiopulmonary bypass: a changing scenario. Ann Thorac Surg. 2015;100:95-100.

32. Lang T, Bauters A, Braun SL, Potzsch B, von Pape KW, Kolde HJ, et al. MultiCentre investigation on reference ranges for ROTEM thromboelastometry. Blood Coagul Fibrinolysis. 2005;16:301-10.

33. Brown JR, Birkmeyer NJ, O'Connor GT. Meta-analysis comparing the effectiveness and adverse outcomes of antifibrinolytic agents in cardiac surgery. Circulation. 2007;115:2801-13.

\section{Publisher's Note}

Springer Nature remains neutral with regard to jurisdictional claims in published maps and institutional affiliations.

Ready to submit your research? Choose BMC and benefit from:

- fast, convenient online submission

- thorough peer review by experienced researchers in your field

- rapid publication on acceptance

- support for research data, including large and complex data types

- gold Open Access which fosters wider collaboration and increased citations

- maximum visibility for your research: over $100 \mathrm{M}$ website views per year

At $\mathrm{BMC}$, research is always in progress.

Learn more biomedcentral.com/submission 